\title{
Efflux Dynamics of the Anti-Epileptic, Levetiracetam, Through the P-Glycoprotein Channel Revealed by Advanced Comparative Molecular Simulations
}

Esmaeil Behmard ( $\sim$ behmard62@gmail.com )

Shiraz University of Medical Sciences

Ebrahim Barzegari

Kermanshah University of Medical Sciences

Sohrab Najafipour

Fasa University of Medical Sciences

Amin Kouhpayeh

Fasa University of Medical Sciences

Younes Ghasemi

Shiraz University of Medical Sciences

Ali A. Asadi-Pooya

Shiraz University of Medical Sciences

Research Article

Keywords:

Posted Date: January 25th, 2022

DOI: https://doi.org/10.21203/rs.3.rs-1263011/v1

License: (9) (i) This work is licensed under a Creative Commons Attribution 4.0 International License.

Read Full License 


\section{Abstract}

Understanding the precise mechanistic details of the possible binding and transport of antiepileptic drugs through the efflux pump, P-glycoprotein (P-gp), is essential to find strategies for the treatment of epileptic patients resistant to anti-seizure medicines. In the present work, conventional molecular dynamics, binding free energy calculations, steered molecular dynamics and umbrella sampling were applied to study the interactions of levetiracetam and brivaracetam with P-gp and their possible egress path from the binding site. Comparative results for the control drugs, zosuquidar and verapamil, confirmed their established P-gp inhibitory activity. Brivaracetam, a non-substrate of P-gp, demonstrated stronger static and dynamic interactions with the exporter protein, than levetiracetam. The potential of mean force calculations indicated that the energy barriers through the ligand export were the lowest for levetiracetam, suggesting the drug as a P-gp substrate with facile passage along the transporter channel. Our findings also stressed the contribution of nonpolar interactions with P-gp channel lining as well as with membrane lipid molecules to hampering the antiepileptic drug efflux by the transmembrane exporter. Appropriate structural engineering of the anti-seizure medications is thus recommended to address the drug-resistant epilepsy.

\section{Introduction}

Epileptic seizures are uncontrollable in about $30-40 \%$ of cases receiving appropriate anti-seizure medications (ASMs) ${ }^{1-3}$. For longer than a century, the epilepsy research community has undertaken the burden of refractory epilepsy through special attention to the discovery of new ASMs or developing the epilepsy surgery techniques. However, the population of patients with drug-resistant epilepsy has not changed conspicuously. Surgeries demonstrate indication for only a limited proportion of refractory epileptic patients, and the success with other introduced methods has been marginal ${ }^{1-3}$. Therefore, investigation to discover new ways and strategies to treat the drug-resistant epilepsy seems to be crucial.

Pharmacokinetic hypothesis and transporter hypothesis are the two most important notions proposed to explain the mechanism of the resistance to ASMs in epileptic disorders ${ }^{4,5}$. A high expression of the membrane transport channel, P-glycoprotein (P-gp), plays a pivotal role in both hypotheses. P-gp has been suggested to prevent its drug substrates from reaching their cellular targets by its efflux pumping function in various tissues, thereby reducing the treatment efficacy.

Though the P-gp export has been established as an underpinning mechanism for resistance to numerous anticancer drugs ${ }^{8}$, research to verify ASMs as substrates for P-gp in the capillary endothelial cells of the blood brain barrier has led to conflicting findings ${ }^{4-7}$. Most of the common anti-epileptic drugs are antagonists of ion channels ${ }^{9}$, which proposes their possibility to also interact with P-gp. Racetam-derived ASMs, such as levetiracetam and its more potent analog, brivaracetam ${ }^{10}$, act by inhibiting N-type highvoltage-activated calcium channels, and also by binding to synaptic vesicle protein $2 \mathrm{~A}$ in the brain ${ }^{9,10}$. Levetiracetam, which is a widely used ASM with a broad therapeutical spectrum against multiple seizure 
types, presents a highlighted example of weak therapeutic response in refractory epilepsies but with unclear capability to undergo the P-gp efflux ${ }^{4-7,11}$. Despite having desirable pharmacological features, some studies have suggested the drug as a probable substrate of $\mathrm{P}-\mathrm{gp}^{4-7}$.

The inconsistent P-gp transportability profile of the ASMs may be attributable to the limitations associated with the experimental methods set up for their investigation ${ }^{5,6}$. In fact, important details on the bio-macromolecular dynamic behavior of the transmembrane channel remain out of reach and obscure when monitored by cell-based assays, animal experiments or the clinical research. Advanced computational modeling methods such as molecular dynamics (MD) simulations, on the other hand, offer a more illuminative study tool, with high temporal resolution recording of the molecular behavior in full atomic detail. To date, corroboratory molecular simulations of the P-gp transport system have been applied for analyzing the interaction of various anticancer agents/inhibitors with P-gp and the lipid bilayer $^{12,13}$, or for proposing dynamic models of the transmission of anticancer drugs through the channel ${ }^{14,15}$.

In the current study, we integrate multiple enhanced process simulation approaches to study the interactions and transport of the ASMs, levetiracetam and brivaracetam, through the outward-facing conformation of human P-gp (hP-gp) to the extracellular space ${ }^{16}$ (Supplementary Fig. S1 online). To have controls with established and remarkably different transport properties, which could also mark the distinction between P-gp inhibitors and substrates $^{8}$, verapamil, a calcium-channel blocker used for ventricular rate control in various diseases and with proven P-gp inhibitory activity, and zosuquidar, an experimental strong P-gp inhibitor, are comparatively studied ${ }^{17-19}$. We also investigate the role of membrane lipid molecules in the drugs' dynamics along the TM channel outwards ${ }^{8}$. The results provide insights into the complete translocation pathway and the dynamic mechanisms involved in the possible ASMs' export by the channel protein, thus helping address the drug-resistance in epilepsies.

\section{Methodology}

Structure preparation and molecular docking of drugs to hP-gp protein. The outward-facing structure of hP-gp was modeled using SWISS-MODEL (Supplementary Fig.

S5 online), (https://swissmodel.expasy.org/) based on the crystal structure $20 \mathrm{NJ}^{16}$. As the initial ligand structures, atom coordinates for zosuquidar, verapamil, brivaracetam and levetiracetam were retrieved from Drug Bank (https://go.drugbank.com/). Hydrogen atoms were added to the ligand structures using OpenBabel (http://www.cheminfo.org). Preparation of protein and ligands structures for molecular docking was carried out in AutoDockFR program ${ }^{23}$. Molecular docking was performed using AutoDock Vina open-source too ${ }^{24}$. For each ligand, the pose with the best binding affinity was selected for further study. To produce 3D diagrams of hydrophobic interactions and hydrogen bonds in the optimal complex of ligands with hP-gp, the PLIP web tool and the open-source molecular visualization software PyMOL were utilized ${ }^{25,26}$. 
Molecular dynamics simulation details. All-atom molecular dynamic simulations were carried out by using the software suite Gromacs-202027. CHARMM-GUI web server (http://www.charmm-gui.org/) was employed for constructing a bilayer membrane model composed of 1-palmitoyl-2-oleoyl-sn-glyucero-3phosphocholine (POPC), and for insertion of the P-gp-drug complexes into the membrane using the replacement method. Then, the system was solvated by adding water molecules to the simulation box. Sodium and chlorine ions were next added to neutralize the system charge and to simulate a physiological salt concentration of $0.15 \mathrm{M}$. The energies and dynamics in all simulation systems were defined by CHARMM36 force field ${ }^{28}$. Periodic boundary conditions were applied in all three dimensions ${ }^{29}$. Topological parameters and atomic point charges for the drug molecules were assigned by the CHARMMGUI web server. Long-range electrostatics were defined using the particle mesh Ewald (PME) method ${ }^{30}$. The cut-off length for both long-range Coulombic bonds and van der Waals (vdW) interactions was set to $1.2 \mathrm{~nm}$. The steepest descent algorithm was applied to perform energy minimizations ${ }^{31}$. To prevent heat shock, the system temperature was gradually increased to reach the desired temperature $(310 \mathrm{~K})$. After that, pressure was applied to the system to achieve the proper density for the system. To avoid the system collapse, NVT and NPT ensembles were implemented with restriction applied on lipids, the protein and the drug. Afterwards, the main simulation was performed for 100000 ps without any restraints.

Binding free energy calculation. Molecular mechanics Poisson-Boltzmann surface area (MM-PBSA) method as implemented in g_mmpbsa program was applied to calculate the energy components in the hP-gp-ligand binding 32 .

Steered molecular dynamics. Because the process of the transport for small molecules through membrane channel proteins occurs on a time scale of milliseconds or seconds, it cannot be studied by conventional simulation methods. Steered molecular dynamics (SMD) addresses this issue by introducing an imaginary external force to the small molecule to drive it through the channel ${ }^{12-15,33,34}$. In the present study, the final structures of the channel-drug complexes obtained from conventional simulations were used as the initial model for performing SMD. Harmonic potential was applied to pull out the drug from the channel; this potential causes the force exerted to the drug to change according to the nature of the connections between the drug and the protein. For this purpose, using the force constant of $1000 \mathrm{~kJ} \cdot \mathrm{mol}^{-1} \cdot \mathrm{nm}^{-2}$, the ligands were hauled out from the binding pocket and moved along the channel at a constant rate of $0.00044 \mathrm{~nm} / \mathrm{ps}$ for $10000 \mathrm{ps}$ SMD. Here, dissociation of the drug from the binding site was considered as the reaction coordinate (RC; $\sim 4.4 \mathrm{~nm}$ ). Other parameters for performing SMD were set according to the conventional simulations. Outputs analyses addressed both drug-channel and druglipid interactions.

Umbrella sampling simulation for drug release. Umbrella sampling simulation is a technique to compute the free energy profile of a chemical reaction, calculated as a potential of mean force (PMF) ${ }^{12-15,33,34}$. This approach involves performing simulations in a series of windows that are extracted from the RC considered in the SMD. At the end of the window series simulation, distributions of samples during the trajectory are combined using the weighted histogram analysis (WHAM) method, and finally an accurate 
PMF curve is calculated along the $\mathrm{RC}^{12-15,33,34}$. In this work, the umbrella sampling approach was adopted to calculate the free-energy profile of the ligands along the hP-gp channel. The $Z$ distance along the RC ( 4.4 nm) was divided into windows every $0.2 \mathrm{~nm}$. Accordingly, the total of 20 windows along the RC were simulated independently, for each ligand. A harmonic umbrella potential with a force constant of $1000 \mathrm{~kJ} . \mathrm{mol}^{-1} \cdot \mathrm{nm}^{-2}$, was applied along the Z-axis. Parrinello-Rahman barostat and Nosé-hoover thermostat were used to maintain the pressure of the system at $1 \mathrm{~atm}$, and the temperature at $310 \mathrm{~K}$, respectively ${ }^{27}$. Each window was equilibrated for 1000 ps followed by 8000 ps of production run. Finally, the WHAM tool was applied to calculate the PMF based on the obtained umbrella sample $12-15,33,34$.

\section{Data availability}

No datasets were generated or analysed during the current study. All data generated or analysed during this study are included in this published article and its Supplementary Information files.

\section{Results}

Stability of the studied systems. To simulate the transport through the hP-gp channel for the ASM, levetiracetam, in comparison with its analogue brivaracetam, the anticancer verapamil, and the P-gp inhibitor zosuquidar, firstly each drug was bound to the efflux protein using the molecular docking approach. Results were indicative of a strong P-gp binding for zosuquidar and verapamil, while the ASMs, particularly levetiracetam, were involved by weak interactions (Tables S1-S4). The systems then underwent conventional MD simulations to provide stable P-gp-drug complexes for the study of ligand transport from the binding site to the extracellular space using SMD and umbrella sampling methods. Figure 1 displays a zoom of the positions of the ligands in the drug binding site of hP-gp after the proteinligand complexes were permitted to relax for $100 \mathrm{~ns}$ of classical MD. Illustration of the residues involving in the interaction with the highest-affinity binding pose of the ligands in the hP-gp channel indicates that non-polar and aromatic residues played the predominant role in the hP-gp-drugs binding (Figure 1A-D and Supplementary Fig. S2 online).

The dynamic stability of the protein in the hP-gp-drug complex, as estimated by the root mean squared deviation (RMSD) parameter of the protein $\mathrm{Ca}$ atoms, indicated that the protein molecule retained its structural stability throughout the simulations (Supplementary Fig. S3A online). To study the dynamic behavior of the drug molecules at the binding pocket during the simulation time, displacement of ligands' heavy atoms relative to their initial position was calculated (Supplementary Fig. S3B online). The results showed that the conformation of the ligands at the binding pocket were very stable and the interactions between the ligands and the residues of the binding site was strong enough to hold the drug molecules in the binding pocket throughout the simulation time (Supplementary Fig. S3B online). These outcomes gave us the assurance that the output was reliable to perform the binding free energy computations respecting the snapshots extracted from the MD trajectory. In addition, the frames produced by MD simulations were reliable enough to contribute to the answer of the questions of ligands' dynamics along the efflux channel. 
Binding energy profile of the drugs. The estimated binding free energy of the pharmaceutical ligands in their interactions with hP-gp, and the share of different energy components in the binding process are listed in Table 1. Based on these results, the favorable component of the free energy tends to be of nonpolar nature. Thus, it can be concluded that hydrophobic interactions are the main driving force of the binding process in the attachment of all studied drugs to hP-gp. Notably, the total free energy and its polar/non-polar contributions indicate a less favorable P-gp binding for levetiracetam and briveracetam in comparison with verapamil and zosuquidar. This is attributable to the smaller size and number of interacting groups on the molecular structure of both ASMs, compared to the P-gp inhibitors (Supplementary Fig. S1 online).

Table 1

The computed binding free energy and contribution of the energy elements $(\mathrm{kJ} / \mathrm{mol})$.

\begin{tabular}{|c|c|c|c|c|}
\hline Energy components & $\begin{array}{l}\text { P-gp/ } \\
\text { Zosuquidar }\end{array}$ & $\begin{array}{l}\text { P-gp/ } \\
\text { Verapamil }\end{array}$ & $\begin{array}{l}\text { P-gp/ } \\
\text { Briveracetam }\end{array}$ & $\begin{array}{l}\text { P-gp/ } \\
\text { Levetiracetam }\end{array}$ \\
\hline Electrostatic energy $\left(\Delta \mathrm{E}_{\text {ele }}\right)$ & 0.786 & 3.062 & 1.119 & -14.477 \\
\hline van der Waals energy $\left(\Delta E_{v d W}\right)$ & -220.232 & -225.565 & -115.521 & -80.863 \\
\hline $\begin{array}{l}\text { Polar solvation energy } \\
\left(\Delta \mathrm{G}_{\text {sol-pol }}\right)\end{array}$ & 90.375 & 109.590 & 74.071 & 53.974 \\
\hline $\begin{array}{l}\text { Non-polar solvation energy } \\
\left(\Delta \mathrm{G}_{\text {sol-np }}\right)\end{array}$ & -22.819 & -27.020 & -14.093 & -11.389 \\
\hline Polar contribution $\left(\Delta \mathrm{E}_{\text {polar }}\right)^{a}$ & 91.161 & 112.652 & 75.19 & 39.497 \\
\hline $\begin{array}{l}\text { Non-polar contribution } \\
\left(\Delta \mathrm{E}_{\text {non-polar }}\right)^{\mathrm{b}}\end{array}$ & -243.051 & -252.585 & -129.614 & -92.222 \\
\hline Binding free energy $\left(\Delta G_{\text {bind }}\right)$ & -151.890 & -139.933 & -54.424 & -52.755 \\
\hline \multicolumn{5}{|l|}{${ }^{\text {a }} \Delta \mathrm{E}_{\text {polar }}=\Delta \mathrm{G}_{\text {sol-pol }}+\Delta \mathrm{E}_{\text {ele }}$} \\
\hline \multicolumn{5}{|l|}{${ }^{\mathrm{b}} \Delta \mathrm{E}_{\text {nonpolar }}=\Delta \mathrm{G}_{\text {sol-np }}+\Delta \mathrm{E}_{\mathrm{vdw}}$} \\
\hline
\end{tabular}

Force fluctuations and energy components during SMD simulations. The SMD-simulated dynamics of the variation of interactions in the process of drug transport along the P-gp channel are illustrated in Figure 2. The initial system and the applied reaction coordinate $(R C)$ can be seen in Figure 2A. The diagram of the force received by ligands moving along the RC during hauler of the drugs from P-gp (Fig. 2B) indicates some local minima in the energy fluctuations in the process of drug's export, which represent favorable Pgp-drug binding geometries along the channel. Pulling the drugs out along the exporter requires the force to be expanded to break the stabilizing bonds, leading to peaks in the SMD force plot (Fig. 2B). 
Binding energies through the SMDs were broken down to short range Coulombic (Coul-SR) and LennardJones (LJ-SR) components to get the electrostatic and vdW contributions, respectively (Fig. 2C-F). The fluctuation patterns of these energies during the SMD were similar to that in the SMD force diagram; i.e. local minima corresponded to larger energy contributions. At the starting points, each drug-P-gp complex is stable with a negative free energy. The energies then decrease and fluctuate during SMD, continuing until all components reach zero, which shows that the drug is completely extruded from the P-gp egress (Fig. 2C-F). Concordantly, the total number of contacts formed between the drugs and hP-gp (Supplementary Fig. S4 online) decreases sharply at about 6000 ps time point of the simulation, for all the drugs. The number of bonds between levetiracetam and the protein is significantly lower than that for the other drugs, and demonstrates a less steep fall with time, which indicates a mild passage and release from the channel.

In all the energy trends, vdW components play the major role in the drug moving process along the P-gp channel (Fig.2C-F). Thus, hydrophobic interactions may be an important agent in thedrug transfer process. The pattern of both total contacts and total interaction energy correspond to the trend of the $\mathrm{vdW}$ energies (Fig. 2C-F). This could emphasize the rate-limiting role of the hydrophobic interactions in the drug efflux through the channel. Therefore, drugs that exhibit more nonpolar interactions with hP-gp are expected to encounter larger energy barriers as they cross the channel, making it more difficult for them to transport out of the cell. Comparatively among the studied drugs, zosuquidar and verapamil showed most, and levetiracetam the least hydrophobic interactions with the lining residues along the Pgp channel. This means that compared to other drugs, zosuquidar barely crosses the P-gp exporter, while levetiracetam crosses the channel easily.

Contribution of lipid molecules to export of ASMs. To clarify the role of lipid molecules of the bilayer membrane in the drugs' transport process, the interaction energies between the drugs and lipids were analyzed in terms of separate electrostatic and vdW components (Fig. 3). The energetic contributions from lipids can be seen to be comparable with hP-gp protein residues. The vdW component has a much greater share than electrostatics in the interaction with drugs during their transmission through hP-gp. In general, the lipid-verapamil interaction was slightly more favorable than the other drugs. The patterns for lipid-zosuquidar and lipid-brivaracetam interactions were almost identical. Among the drugs, levetiracetam showed the least affinity to bind to lipid molecules. The potency of the drugs' interaction with lipid molecules correlates well with that for binding to hP-gp, and signifies the difficulty of the transport of the drugs accordingly. It can be said that the combined connections from hP-gp and lipid molecules work together to prevent the transport of the drugs.

Lining of the passageway for the ASMs. In order to study the roles of residues in the drugs' moving process along the P-gp channel during the SMD simulation, the dynamic P-gp-drug interactions were analyzed, and revealed a set of mainly hydrophobic residues interacting with the drugs as they passed through the channel (Fig. 4). These included for zosuquidar (L65, M69, F332, F335, F336, F343, Q347), verapamil (L65, M69, F303, F336, L339), brivaracetam (L65, M69, Q347), and levetiracetam (L65, M68, M69, F332, F335, L339, F343). SMD energies for individual lining residues highlighted the role of vdW 
forces in their interactions with the drugs. Zosuquidar and brivaracetam, both proved as highly potent Pgp binders, indicated also a favorable electrostatic binding to Q347 of P-gp.

Important bindings appear as local and global minima in the energy plots (Fig. 4). The deeper the local minimum a ligand gets stuck in while dynamically interacting with the P-gp transport passageway, the harder it will cross the channel. Thus, deep minima indicate the energy barriers present in the efflux path of the drug. There is an inverse relationship between the ease of drug's movement in the P-gp channel and the strength of its interaction with lining residues. Our findings show that stronger P-gp-drug interactions in the inner vestibule occur for zosuquidar and verapamil, compared to levetiracetam and brivaracetam. The negative energy values for zosuquidar and verapamil often exceed $-20 \mathrm{~kJ} / \mathrm{mol}$, but they are frequently less than $-20 \mathrm{~kJ} / \mathrm{mol}$ for levetiracetam and even less than $-5 \mathrm{~kJ} / \mathrm{mol}$ for brivaracetam (though the latter is favored by a strong ionic binding to Q347). These interactions create a larger energy barrier against the passage of the inhibitors through the P-gp channel, while the ASM, levetiracetam, experiences a facile passage along its specific lining in the channel.

Potential of Mean Force from Umbrella Sampling. To gain a deeper understanding of the dynamic drugresidue interactions along the hP-gp channel, the energy fluctuations relevant to drugs' transmembrane export during SMD were calculated through the umbrella sampling technique (Fig. 5). The computed potential of mean force (PMF) of drugs through the channel is presented in Fig. 5P, and structurally analyzed in the associated panels for zosuquidar (Fig. 5Q), verapamil (Fig. 5R), brivaracetam (Fig. 5S), and levetiracetam (Fig. 5T).

According to Figure $5 \mathrm{Q}(\mathrm{a}-\mathrm{d})$, when zosuquidar moves along the P-gp channel, various hydrophobic interactions and a halogen bond occur between the drug and the lining residues. As a result, the PMF diagram increases steadily with a sharp slope (0.3-3.66 nm of RC; Fig. 5P), then achieves to a plateau at $3.66 \mathrm{~nm}$ (Fig. 5P), meaning that the compound is completely extruded from the channel. A maximum energy barrier of about $210 \mathrm{~kJ} / \mathrm{mol}$ is encountered along the drug's passage through the inner vestibule of the exporter. At the beginning of the transport process, the PMF diagram of verapamil reduces from 0 to $-12.27 \mathrm{~kJ} / \mathrm{mol}$, then the molecule is trapped into its main minimum at $\mathrm{RC} \approx 0.25 \mathrm{~nm}$ (Fig. 5P and $5 \mathrm{R}(\mathrm{a})$ ). To break this barrier, the drug must ascend to $a \approx 92.12 \mathrm{~kJ} / \mathrm{mol}$ of energy level (Fig. 5P and $5 \mathrm{R}(\mathrm{a}-\mathrm{b})$ ). At this point, the system first breaks up the hydrophobic interactions between verapamil and the involving lining residues (L65, L126, A129, I299, F303, L339, A342 and F343) (Fig. 5R(a-b)); thus, PMF value increases relatively sharply until RC of $1.67 \mathrm{~nm}$. Then, the trend becomes smooth (Fig. 5P and 5R(c-d)). At the RC of $3.62 \mathrm{~nm}$, the PMF of verapamil reaches a plateau, meaning that the drug is entirely detached from the P-gp protein (Fig. 5P).

In the diagram for brivaracetam, two different phases can be seen during the transport process (Fig. 5P). A rapid increase phase from 0.1 to $0.84 \mathrm{~nm}$ (Fig. 5P and 5S(a)), and a steady slower increase phase from 0.84 to $2.7 \mathrm{~nm}$ (Fig. 5P and 5S(b-d)). In the rapid increase phase of brivaracetam release, the hydrophobic interactions between the drug and some residues (V133, W136, and 1190) are broken (Fig. 5S(a)); as a result, the value of the PMF increases rapidly (Fig. 5P). The next phase represents the breakage of bonds 
between the residues E74, V133, Q132, G191, Q132, Q195, Q347 and P350 of the P-gp channel, and brivaracetam during the umbrella sampling simulation time (Fig. 5P and Fig. 5S(b-d)). Thus, the PMF value gradually increases between points $0.84 \mathrm{~nm}$ and $2.7 \mathrm{~nm}$ (Fig. 5P). The PMF diagram of brivaracetam reached plateaus, suggesting the entirely release of the drug from the P-gp transporter protein (Fig. 5P).

It can be seen from the PMF diagram of levetiracetam that the energy is gradually increased from -3.13 to the barrier of $12.12 \mathrm{~kJ} / \mathrm{mol}$ (Fig. 5P and $5 \mathrm{~T}(\mathrm{a}-\mathrm{b})$ ), after which it falls into its global minimum (Fig. 5P and $5 \mathrm{~T}(\mathrm{~b}) ; \mathrm{RC} \approx 0.69 \mathrm{~nm})$. Obviously, there are hydrophobic and electrostatic interactions between levetiracetam and lining residues in this area (Fig. 5T(b)). Therefore, the inner vestibule of the P-gp channel has the highest potency to capture levetiracetam; hence, we suggest that this area is the binding site of levetiracetam in the hP-gp channel (Fig. 5T(b)). According to the PMF diagram, levetiracetam encounters an energy barrier of about $83.2 \mathrm{~kJ} / \mathrm{mol}$ while crossing the channel (Fig. $5 \mathrm{P}, \mathrm{RC} \approx 2 \mathrm{~nm}$, and 5T(b-d)). Therefore, at the beginning of the transport process, the PMF trend of levetiracetam rises rapidly to pass the energy barrier (Fig. $5 \mathrm{P}$ and $5 \mathrm{~T}(\mathrm{~b}-\mathrm{d}), \mathrm{RC} \approx 0.69-2 \mathrm{~nm}$ ). After the drug passes through this area $(\mathrm{RC} \approx 2 \mathrm{~nm})$, the diagram gradually reaches plateaus, meaning that the drug has moved away and extruded from the channel (Fig. 5P).

\section{Discussion}

More than one-third of patients with epilepsy demonstrate resistance to ASMs. Failing to assimilate the drug in the intestine or reduced drug uptake at the blood-brain barrier could limit the therapeutic response to drugs. Hypothetically, drug transport through efflux transporters, such as P-gp, plays a significant role in this drug resistance. In the present work, MD and SMD followed by umbrella sampling methods were applied to study the transport behaviors of the ASMs, levetiracetam and brivaracetam, comparatively with zosuquidar and verapamil, along the human P-gp channel. At the start, molecular dockings revealed the lower affinity of levetiracetam for binding to P-gp, than other studies compounds, which suggested it as a P-gp substrate, because the rate of drug transport increases exponentially with decreasing affinity to the transporter ${ }^{17}$. The results of advanced simulations also clearly showed that levetiracetam could be easily transported out of the cytosol through the hP-gp channel. These findings strongly resonate the previous clinical observations that P-gp function may play a significant role in drug-resistant epilepsy ${ }^{4-7}$.

According to SMD PMFs, the free energy barrier for the transfer of zosuquidar, known as a specific hP-gp inhibitor, from the hP-gp channel was shown to be much larger than that for verapamil, a P-gp antagonist anticancer; thus, the transfer of the former through the channel is much more difficult than the latter. This result is in strong agreement with experimental observations, showing zosuquidar $\left(\Delta \mathrm{G}_{\text {unbind }} \approx-210\right.$ $\mathrm{kJ} / \mathrm{mol})$ as a more effective P-gp inhibitor than verapamil $\left(\Delta \mathrm{G}_{\text {unbind }} \approx-152.36 \mathrm{~kJ} / \mathrm{mol}\right)^{18,19}$. In like manner, the PMF energy barriers for levetiracetam passing from P-gp were significantly lower than that of the other drugs, showing a readily export of the ASM drug by the channel. As a result, levetiracetam can be the substrate of hP-gp, which has been suggested in some experimental studies ${ }^{4-7}$. Additionally, the 
higher free energy barriers of brivaracetam than levetiracetam suggests that this ASM ligand may transport more difficultly by the channel. Practical research shows that brivaracetam is not a substrate of hP-gp ${ }^{20}$; thus, our computational results are consistent with experimental observations.

Noteworthily, the role of lipid molecules from the bilayer membrane was incorporated in the study of the drug export by P-gp, in addition to the contribution of the channel lining residues ${ }^{8}$. Hydrophobic interactions of the drugs with the inner vestibule regions of P-gp and the membrane lipid molecules increased the energy barriers of the drug transport through hP-gp, consequently restricting the drug passing through the channel. Zosuquidar and verapamil demonstrated the most hydrophobic interactions with hP-gp as well as lipid molecules, compared to other drugs. On the contrary, the PMF fluctuations from umbrella sampling revealed that levetiracetam, which had the weakest hydrophobic interactions with hP-gp as well as lipid molecules among the studied drugs, encountered a smaller energy barrier on its passage through hP-gp and was gently transported by the channel protein. Therefore, decreased vdW interactions between the drug and hP-gp as well as lipid molecules is beneficial to the drug transport process. These findings are strongly consistent with experimental observations. As an example,

hydrophobic progesterone was shown to bind to, but did not appear to be transported by, P-gp ${ }^{21}$. In fact, despite being a potent steroid transporter, P-gp does transport hydrophilic steroids more readily than hydrophobic ones; as a result, these steroids may not be good P-gp inhibitors, while most lipophilic steroids can act as strong channel antagonists ${ }^{21}$. Another study reported that antifolate derivatives with more nonpolar nature showed more suppressing activity against resistant tumor cells, than those with more polar groups, because those derivatives less tended to be transported by P-gp 22 .

\section{Conclusion}

The important ASM, levetiracetam, was shown to easily cross the hP-gp channel, when compared to other control drugs. The facile passage of this antiepileptic drug through its specific lining in the channel supports its consideration as a P-gp substrate. From the analyses of interaction energies between the drugs and the channel as well as lipid molecules through steered dynamic modeling, it can be hypothesized that vdW energies are the main driving force in hindering the efflux of drugs by the human P-gp transport channel. Based on the correlation between our computational results and previous experimental observations, our study provides insights to cope with the ASM drug resistance in refractory epilepsies by manipulation of the hydrophobic interactions between the pharmaceutical compound and both P-gp and membrane lipids. Furthermore, it provides a rational theoretical basis to determining whether a compound acts as a substrate or inhibitor in relation to the hP-gp channel.

\section{Declarations}

\section{Author Contributions}

E.B. (First author) performed data curation, analysis, validation and visualization; and wrote the original draft. E.B. (Second author) contributed in conceptualized the work, defined methodology and completing 
the draft. S.N. contributed in conceptualized the work and writing the original draft. A.K. contributed in conceptualized the work and writing the original draft. Y.G. contributed in project administration, resources and funding acquisition. A.A.A. contributed in project administration, supervision. All authors reviewed the final manuscript.

\section{Competing Interests Statement}

The authors declare no competing interests.

\section{Additional Information}

Supplementary information accompanies this paper, including Supplementary Tables S1-S4 and Figures S1-S5.

\section{References}

1. Ryvlin, P. (2005). The modern challenges of drug resistant epilepsy. Epileptic Disorders, 7(1), 1-2.

2. Mesraoua, B., Deleu, D., Kullmann, D. M., Shetty, A. K., Boon, P., Perucca, E., ... \& Asadi-Pooya, A. A. (2019). Novel therapies for epilepsy in the pipeline. Epilepsy \& Behavior, 97, 282-290. DOI: 1016/j.yebeh.2019.04.042

3. Beghi, E. (2020). The epidemiology of epilepsy. Neuroepidemiology, 54(2), 185-191. DOI: $10.1159 / 000503831$

4. Tang, F., Hartz, A., \& Bauer, B. (2017). Drug-resistant epilepsy: multiple hypotheses, few answers. Frontiers in neurology, 8, 301. DOI:3389/fneur.2017.00301.

5. Zhang, C., Kwan, P., Zuo, Z., \& Baum, L. (2012). The transport of antiepileptic drugs by P-glycoprotein. Advanced drug delivery reviews, 64(10), 930-942. DOI: 1016/j.addr.2011.12.003

6. Lee, G. H., Kim, B. M., Kang, J. K., \& Lee, S. A. (2013). Loss of the initial efficacy of levetiracetam in patients with refractory epilepsy. Seizure, 22(3), 185-188. DOI:1016/j.seizure.2012.12.002.

7. Luna-Tortós, C., Fedrowitz, M., \& Löscher, W. (2008). Several major antiepileptic drugs are substrates for human P-glycoprotein. Neuropharmacology, 55(8), 1364-1375.

DOI:1016/j.neuropharm.2008.08.032.

8. Seelig, A. (2020). P-Glycoprotein: One Mechanism, Many Tasks and the Consequences for Pharmacotherapy of Cancers.Frontiers in Oncology, 10. DOI:3389/fonc.2020.576559

9. Aneja, S., \& Sharma, S. (2013). Newer anti-epileptic drugs.Indian Pediatrics, 50(11), 1033-1040. DOI:1007/s13312-013-0284-9

10. Mastroianni, G., Ascoli, M., Gasparini, S., Brigo, F., Cianci, V., Neri, S., ... \& Ferlazzo, E. (2021). Therapeutic approach to difficult-to-treat typical absences and related epilepsy syndromes. Expert review of clinical pharmacology, 14(11), 1427-1433. DOI:1080/17512433.2021.1959317

11. Asadi-Pooya, A. A., \& Sperling, M. R. (2016). Antiepileptic drugs: a clinician's manual. Oxford University Press. 
12. Zhang, D. Li, T. Sun, L. Liang, Q. Wang, Interaction of P-glycoprotein with anti-tumor drugs: the site, gate and pathway, Soft Matter. 11 (2015)6633-41. DOI:10.1039/C5SM01028D.

13. Zhang, J., Sun, T., Liang, L., Wu, T., \& Wang, Q. (2014). Drug promiscuity of P-glycoprotein and its mechanism of interaction with paclitaxel and doxorubicin. Soft Matter, 10(3), 438-445. DOI:1039/C3SM52499J.

14. McCormick, J. W., Vogel, P. D., \& Wise, J. G. (2015). Multiple drug transport pathways through human P-glycoprotein. Biochemistry, 54(28), 4374-4390. DOl:1021/acs.biochem.5b00018.

15. Ferreira, R. J.; Ferreira, M.-J. U.; dos Santos, D. J. V. A. Insights on P-glycoprotein's efflux mechanism obtained by molecular dynamics simulations. J Chem Theory Comput 2012, 8, 1853-1864. DOI:1021/ct300083m

16. Dawson, R. J., \& Locher, K. P. (2007). Structure of the multidrug ABC transporter Sav1866 from Staphylococcus aureus in complex with

17. Äänismaa, P., \& Seelig, A. (2007). P-Glycoprotein kinetics measured in plasma membrane vesicles and living cells. Biochemistry, 46(11), 3394-3404. DOI: 10.1021/bi0619526.

18. Syed, S. B., Arya, H., Fu, I. H., Yeh, T. K., Periyasamy, L., Hsieh, H. P., \& Coumar, M. S. (2017). Targeting P-glycoprotein: Investigation of piperine analogs for overcoming drug resistance in cancer. Scientific reports, 7(1), 1-18. DOI:10.1038/s41598-017-08062-2

19. Shukla, S., Abel, B., Chufan, E. E., \& Ambudkar, S. V. (2017). Effects of a detergent micelle environment on P-glycoprotein (ABCB1)-ligand interactions. Journal of Biological Chemistry, 292(17), 7066-7076. DOI: 10.1074/jbc.M116.771634

20. Rizzo, A., Donzelli, S., Girgenti, V., Sacconi, A., Vasco, C., Salmaggi, A., ... \& Ciusani, E. (2017). In vitro antineoplastic effects of brivaracetam and lacosamide on human glioma cells. Journal of Experimental \& Clinical Cancer Research, 36(1), 1-13. DOI: 10.1186/s13046-017-0546-9

21. Wang, R. B., Kuo, C. L., Lien, L. L., \& Lien, E. J. (2003). Structure-activity relationship: analyses of pglycoprotein substrates and inhibitors. Journal of clinical pharmacy and therapeutics, 28(3), 203228. DOI: 10.1046/j.1365-2710.2003.00487.x.

22. Selassie, C. D., Strong, C. D., Hansch, C., Delcamp, T. J., Freisheim, J. H., \& Khwaja, T. A. (1986). Comparison of triazines as inhibitors of $\mathrm{L} 1210$ dihydrofolate reductase and of $\mathrm{L} 1210$ cells sensitive and resistant to methotrexate. Cancer research, 46(2), 744-756.

23. Ravindranath, P. A., Forli, S., Goodsell, D. S., Olson, A. J., \& Sanner, M. F. (2015). AutoDockFR: advances in protein-ligand docking with explicitly specified binding site flexibility. PLoS computational biology, 11(12), e1004586. DOI: 10.1371/journal.pcbi.1004586.

24. Trott, O., \& Olson, A. J. (2010). AutoDock Vina: improving the speed and accuracy of docking with a new scoring function, efficient optimization, and multithreading. Journal of computational chemistry, 31(2), 455-461. DOI:10.1002/jcc.21334.

25. Adasme, M. F., Linnemann, K. L., Bolz, S. N., Kaiser, F., Salentin, S., Haupt, V. J., \& Schroeder, M. (2021). PLIP 2021: Expanding the scope of the protein-ligand interaction profiler to DNA and RNA. 
Nucleic Acids Res. DOI:1093/nar/gkab294.

26. DeLano, W. L. (2002). The PyMOL molecular graphics system. http://www.pymol.org.

27. Van Der Spoel, D., Lindahl, E., Hess, B., Groenhof, G., Mark, A. E., \& Berendsen, H. J. (2005). GROMACS: fast, flexible, and free. Journal of computational chemistry, 26(16), 1701-1718. DOI:1002/jcc.20291.

28. Vanommeslaeghe, K., Hatcher, E., Acharya, C., Kundu, S., Zhong, S., Shim, J., ... \& Mackerell Jr, A. D. (2010). CHARMM general force field: A force field for drug-like molecules compatible with the CHARMM all-atom additive biological force fields. Journal of computational chemistry, 31(4), 671690. DOI:1002/jcc.21367.

29. Makov, G., \& Payne, M. C. (1995). Periodic boundary conditions in ab initio calculations. Physical Review B, 51(7), 4014. DOl:1103/PhysRevB.51.4014.

30. Essmann, U., Perera, L., Berkowitz, M. L., Darden, T., Lee, H., \& Pedersen, L. G. (1995). A smooth particle mesh Ewald method. The Journal of chemical physics, 103(19), 8577-8593. DOI:1063/1.470117.

31. Fliege, J., \& Svaiter, B. F. (2000). Steepest descent methods for multicriteria optimization. Mathematical methods of operations research, 51(3), 479-494. DOI: 1007/s001860000043.

32. Kumari, R., Kumar, R., Open Source Drug Discovery Consortium, \& Lynn, A. (2014). g_mmpbsa: A GROMACS tool for high-throughput MM-PBSA calculations. Journal of chemical information and modeling, 54(7), 1951-1962. DOI: 1021/ci500020m.

33. Ferreira, R. J.; Ferreira, M.-J. U.; dos Santos, D. J. V. A. Do drugs have access to the P-glycoprotein drug-binding pocket through gates? J Chem Theory Comput 2015, 11, 4525-4529.

DOI:1021/acs.jctc.5b00652

34. Subramanian, N.; Condic-Jurkic, K.; Mark, A. E.; O'Mara, M. L. Identification of possible binding sites for morphine and nicardipine on the multidrug transporter P-glycoprotein using umbrella sampling techniques. J Chem Inf Model 2015, 55, 1202-1217. DOI:1021/ci5007382

\section{Figures}

\section{Figure 1}

Human P-gp complex with (A) zosuquidar , (B) verapamil, (C) brivaracetam, and (D) levetiracetam, after molecular dynamics simulations. hP-gp is represented as light blue cartoon, and ligands as wheat sticks. The key residues (blue sticks) are labeled. Hydrogen bonds are shown as blue line, and hydrophobic interactions in red dots. 
(A) Structure of P-gp in the bilayer membrane at the beginning of SMD, and different binding modes of a drug moving along the channel. (B) Force changes during the drug transport process through the SMD simulation. (C-F) Graphs of the SMD energies between P-gp and respectively zosuiquidar, verapamil, briveracetam and levetiracetam, indicating the short-range Coulombic interactions (yellow), short-range Lennard-Jones interactions (red), and the total interaction energy (blue).

\section{Figure 3}

(A) van der Waals, and (B) electrostatic energies for the interactions of drugs with membrane bilayer lipids.

\section{Figure 4}

Energy profiles for the key lining residues of the P-gp transport channel in the interaction with studied drugs, showing the van der Waals (black) and electrostatic (red) energy components.

\section{Figure 5}

(P) The PMF profile change for the transport of zosuquidar, verapamil, brivaracetam and levetiracetam through the hP-gp channel. The a-d tags represent the main intermediate structures of hP-gp, whose snapshots are illustrated in the panels Q-T for the drugs, respectively. (Q-T) Interactions between the drugs and the inner residues of the main intermediate structures of hP-gp channel during umbrella sampling simulations. Drugs are shown as wheat sticks. The lining residues (blue sticks) are labeled. Hydrogen bonds are shown as blue lines, hydrophobic interactions as red dots, and the halogen bond as a green line.

\section{Supplementary Files}

This is a list of supplementary files associated with this preprint. Click to download.

- Supportinginformation.docx 